\title{
Separation of radiation from two sources from their known radiated sum field
}

\author{
Laitinen, Tommi; Pivnenko, Sergey
}

Published in:

2011 XXXth URSI General Assembly and Scientific Symposium

Link to article, DOI:

10.1109/URSIGASS.2011.6050250

Publication date:

2011

Document Version

Early version, also known as pre-print

Link back to DTU Orbit

Citation $(A P A)$ :

Laitinen, T., \& Pivnenko, S. (2011). Separation of radiation from two sources from their known radiated sum field. In 2011 XXXth URSI General Assembly and Scientific Symposium

https://doi.org/10.1109/URSIGASS.2011.6050250

\section{General rights}

Copyright and moral rights for the publications made accessible in the public portal are retained by the authors and/or other copyright owners and it is a condition of accessing publications that users recognise and abide by the legal requirements associated with these rights.

- Users may download and print one copy of any publication from the public portal for the purpose of private study or research.

- You may not further distribute the material or use it for any profit-making activity or commercial gain

- You may freely distribute the URL identifying the publication in the public portal

If you believe that this document breaches copyright please contact us providing details, and we will remove access to the work immediately and investigate your claim. 


\title{
Separation of radiation from two sources from their known radiated sum field
}

\author{
$\underline{\text { Tommi Laitinen }}^{1}$ and Sergey Pivnenko ${ }^{2}$ \\ ${ }^{1}$ Aalto University School of Electrical Engineering, Department of Radio Science and Engineering \\ P.O. Box 13000, 00076 AALTO, Finland, Tommi.Laitinen@aalto.fi \\ ${ }^{2}$ Department of Electrical Engineering, Technical University of Denmark \\ DK-2800, Kgs. Lyngby, Denmark, sp@elektro.dtu.dk
}

\begin{abstract}
This paper presents a technique for complete and exact separation of the radiated fields of two sources (at the same frequency) from the knowledge of their radiated sum field. The two sources can be arbitrary but it must be possible to enclose the sources inside their own non-intersecting minimum spheres so that the closest distance between the surfaces of the two spheres is of the order of at least a few wavelengths.
\end{abstract}

\section{Introduction}

Development of tools for analysis of the field radiated by antennas and for antenna diagnostics has fascinated researchers over the years. In [1], a diagnostics technique, for example, for detecting a faulty element of an antenna array from the known spherical radiation pattern has been developed. This method can be used to produce a picture of fields on the surface of an antenna array, and this picture then provides knowledge of the functioning of the array, for example, by visual inspection. As well, attempts for separating the radiated field of the actual antenna element from the field scattered from the surroundings based on the known spherical radiation pattern have been made [2]. In this technique, the radiation pattern is expanded into spherical waves, and the part of the spherical wave spectrum that can be considered not to evolve from the antenna element is filtered out. Although the technique in [2] does separate part of the radiation caused by the scattering from the surrounding structures from that of the element radiation, some part of the radiation desired to be filtered out is presented by the same spherical modes that are used for characterizing the radiation of the element. Hence, the element radiation cannot be completely separated from that of the surroundings.

The purpose of this paper is to present a technique for complete and exact separation of the radiated fields from two arbitrary sources from the knowledge of their sum radiation pattern. The two sources can be arbitrary but it must be possible to enclose the sources inside their own non-intersecting minimum spheres so that the closest distance between the surfaces of the two spheres is of the order of at least a few wavelengths.

\section{Theory}

The radiation pattern of any radiating structure outside its minimum sphere can be characterized with a truncated spherical wave expansion

$$
\mathbf{E}(r, \theta, \phi)=\frac{k}{\sqrt{\eta}} \sum_{s=1}^{2} \sum_{n=1}^{N} \sum_{m=-n}^{n} Q_{s m n} \mathbf{F}_{s m n}^{(3)}(r, \theta, \phi),
$$

where $\mathbf{E}(r, \theta, \phi)$ is the radiated electric field in spherical coordinates $(r, \theta, \phi), \eta$ is the admittance of the medium, $k$ is the wavenumber, $N$ is the truncation number for the $n$ index of the expansion, $Q_{s m n}$ are the spherical vector wave coefficients, and $\mathbf{F}_{s m n}^{(3)}(r, \theta, \phi)$ are the power-normalized spherical vector wave functions. The well-known truncation rule, according to which the $N$ can be defined, is

$$
N=\left[k r_{0}\right]+n_{1}
$$

Here $k$ is the wavenumber and $r_{0}$ is the radius of the minimum sphere of the radiating source. The $n_{1}$ depends on the accuracy criterion in the field characterization with the spherical wave expansion and typically $n_{1}=10$ to 15 is sufficient for most practical purposes. 
Suppose now that we have a radiating structure consisting of two radiating sources separated in space so that each of these radiating sources can be enclosed inside their minimum spheres according to Fig. 1 and that the surfaces of these minimum spheres do not intersect. Free space conditions outside the two minimum spheres are assumed. Both sources are radiating at the same, fixed frequency. This situation could occur, for example, in such cases where there is a well-defined scatterer nearby a radiating element or two sources are intentionally excited with the same signal. Suppose then that the radiation pattern of the complete structure, that is the sum pattern of the two sources, is known, for example, from measurements, but the radiation patterns of the individual sources are unknown. We define the unprimed $(x, y, z)$ coordinate system, such that the center of the minimum sphere of source 1 is located in its origin, and the primed $\left(x^{\prime}, y^{\prime}, z^{\prime}\right)$ coordinate system, such that the center of minimum sphere of source 2 is located in its origin. The $z$ and $z$ ' axes are coincident. The separation distance $d$ is the distance between the centers of the coordinate systems. The radii of the two minimum spheres is $r_{0}$ and $r_{0}{ }^{\prime}\left(d>r_{0}+r_{0}{ }^{\prime}\right)$. In this paper $r_{0}=r_{0}$ ' is assumed for simplicity, but it is noted that the underlying theory could be also formulated for the case where this assumption is not made.

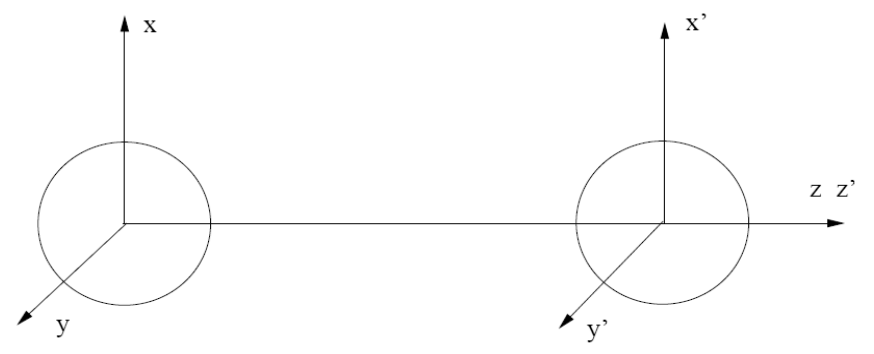

Fig. 1. Illustration of the minimum spheres of two radiating sources.

We now consider the radiation of sources 1 and 2 and their sum radiation in terms of the spherical wave expansion (1). Reorganizing the summations in (1), the electric field of source 1 in the unprimed $(r, \theta, \phi)$ coordinate system and that of the source 2 in the primed $\left(r^{\prime}, \theta^{\prime}, \phi^{\prime}\right)$ coordinate system can be written as

$$
\begin{gathered}
\mathbf{E}_{1}(r, \theta, \phi)=\frac{k}{\sqrt{\eta}} \sum_{m=-N_{1}}^{N_{1}} \sum_{n=n_{0}}^{N_{1}} \sum_{s=1}^{2} Q_{s m n}^{(1)} \mathbf{F}_{s m n}^{(3)}(r, \theta, \phi), \\
\mathbf{E}_{2}\left(r^{\prime}, \theta^{\prime}, \phi^{\prime}\right)=\frac{k}{\sqrt{\eta}} \sum_{m=-N_{1}}^{N_{1}} \sum_{n=n_{0}}^{N_{1}} \sum_{s=1}^{2} Q_{s m n}^{(2)} \mathbf{F}_{s m n}^{(3)}\left(r^{\prime}, \theta^{\prime}, \phi^{\prime}\right),
\end{gathered}
$$

respectively. Here $Q_{s m n}^{(1)}$ and $Q_{s m n}^{(2)}$ are the spherical vector wave coefficients of the radiated fields of sources 1 and 2 in the unprimed and primed coordinate systems, respectively, and $n_{0}=\max (1,|m|)$. According to (2), the truncation number $N_{1}$ can be assumed the same for both expansions as $r_{0}=r_{0}$ ' holds. The radiated field of source 2 in the unprimed coordinate system (for $r>d+r_{0}$ ') is next expressed as

$$
\mathbf{E}_{2}(r, \theta, \phi)=\frac{k}{\sqrt{\eta}} \sum_{m=-N}^{N} \sum_{n=n_{0}}^{N} \sum_{s=1}^{2} Q_{s m n}^{(2)} \mathbf{F}_{s m n}^{(3)}(r, \theta, \phi),
$$

where $Q_{s m n}^{(2)}$ are the spherical vector wave coefficients of the radiated field of source 2 in the unprimed coordinate system. The truncation number $N$ is here obtained from (2) using $r_{0}=d+r_{0}$, and hence $N$ is naturally greater than $N_{1}$ in (3) and (4).

It is known from the translation theorem for the spherical vector wave functions that the radiation of a spherical wave mode with the phase reference point in one coordinate location may be expressed as a weighted sum of spherical wave modes with the phase reference point in another coordinate location [3]. In the situation depicted in Fig. 1, the spherical vector wave coefficients of the radiated field of source 2 in the unprimed coordinate system, $Q_{s m n}^{(2)}$, can be related to those in the primed coordinate system, $Q_{s m n}^{\prime(2)}$. Due to the fact that the translation is in the direction of the $z$ and $z^{\prime}$ axes, and that these axes are coincident, this relation can be written conveniently separately for each fixed $m$ index in the matrix form as

$$
\mathbf{q}_{m}^{(2)}=\mathbf{C}_{m} \mathbf{q}_{m}^{\prime(2)},
$$


which in its expanded form is

$$
\left[\begin{array}{c}
Q_{1 m n_{0}}^{(2)} \\
\vdots \\
Q_{2 m N}^{(2)}
\end{array}\right]=\left[\begin{array}{ccc}
c_{m, 11} & \cdots & c_{m, 1 K^{\prime}} \\
& & \\
\vdots & \ddots & \vdots \\
& & \\
c_{m, K 1} & \cdots & c_{m, K K^{\prime}}
\end{array}\right] \cdot\left[\begin{array}{c}
Q_{1 m n_{0}}^{(2)} \\
\vdots \\
Q_{2 m N_{1}}^{\prime(2)}
\end{array}\right]
$$

Here, the matrix $\mathbf{C}_{m}$ linearly relates the spherical vector wave coefficients of the field of source 2 in the unprimed and primed coordinate systems. The coefficients are obtainable from the knowledge of the translation distance $d$ in wavelengths [3].

We now expand the total radiated field into spherical waves using (3)-(7) and obtain

$$
\mathbf{E}_{t o t}(r, \theta, \phi)=\frac{k}{\sqrt{\eta}} \sum_{m=-N_{1}}^{N_{1}} \sum_{n=n_{0}}^{N_{1}} \sum_{s=1}^{2}\left(Q_{s m n}^{(1)}+Q_{s m n}^{(2)}\right) \mathbf{F}_{s m n}^{(3)}(r, \theta, \phi)+\frac{k}{\sqrt{\eta}} \sum_{m=-N_{1}}^{N_{1}} \sum_{n=N_{1}+1}^{N} \sum_{s=1}^{2} Q_{s m n}^{(2)} \mathbf{F}_{s m n}^{(3)}(r, \theta, \phi),
$$

where, importantly, the weights in the right-most expansion, where the $n$ summation is the for $n=N_{1}+1$ to $N$, comprise only the coefficients $Q_{s m n}^{(2)}$. Hence, as the radiated total field is known, we have also the coefficients $Q_{s m n}^{(2)}$ of source 2 for $n=N_{1}+1$ to $N$ available from the spherical wave expansion of the total field. We may now form a new matrix equation from the lower part of the equation (7) for the coefficients $Q_{s m n}^{(2)}$ as a function of coefficients $Q_{s m n}^{(2)}$ for the indices $n=N_{1}$ +1 to $N$ only. Hence, the upper part of the matrix equation for the indices $n=1$ to $N_{1}$ is left unexploited. It is noted that although we are not using the information on $Q_{s m n}^{(2)}$ for $n=1 \ldots N_{1}$, we may still be able to find the $Q_{s m n}^{\prime(2)}$ for $n=1 \ldots N_{1}$ from (7). Provided the condition number of this matrix equation is sufficiently low, this enables us to find all $Q_{s m n}^{(2)}$ for $m=N_{1} \ldots N_{1}$ for $n=n_{0} \ldots N_{1}$ for $\mathrm{s}=1$ and 2, and finally obtain the radiated field of source 2 from (4). Hence, in this way, the radiation from source 2 can be found and can be separated from that of source 1 . In practice, this is done by recalculating the $Q_{s m n}^{(2)}$ for all $n=1 \ldots N$ from (7) and then calculating the field of source 2 in the unprimed coordinate system from (5), and finally subtracting this field from the known sum field.

\section{Calculation example}

The above theory is tested with computer calculations in this paper. Source 1 is a $0.75 \lambda \times 0.75 \lambda \times 0.75$ $\lambda$ cubically shaped source region centered in the origin of the unprimed coordinate system. This cubical source region consists of 64 separate coordinate locations within $\lambda / 2$ interval in $x, y$ and $z$, so that in each of coordinate locations an $x$, $y$ and $z$-polarized electric and an $x, y$ and $z$-polarized magnetic Hertzian dipole is located. The excitation coefficient of each dipole is random, and hence, generally different. This complex excitation coefficient is $\alpha \exp (\mathrm{j} 2 \pi \beta)$, where both $\alpha$ and $\beta$ are random numbers between 0 and 1 . Source 2 is a similar source, but it is centered at the distance of approximately $d=8.15 \lambda$ in the direction of $+\mathrm{z}$ axis in the origin of the primed coordinate system. The $r_{0}=r_{0}{ }^{\prime} \approx 1.3 \lambda$. The situation is comparable to that shown in Fig. 1. The truncation number $N_{1}=15$ to be applied in (3)-(4) and $N=71$ to be applied in (5) are obtained from (2) using $n_{1}=6$ and 14 , respectively.

The far fields of sources 1 and 2 and their sum far field are calculated in the unprimed coordinate system. The spherical $n$-mode spectra of these three fields are determined and these spectra are shown in Fig. 2 (a)-(c). The spectra clearly show that $N_{1}=15$ is sufficient for characterizing the radiation of source 1 in the unprimed coordinate system, and $N=71$ is sufficient for characterizing the radiation of source 2 in the unprimed coordinate system. The spectra give a clear indication that a simple filtering of the modes with $n>15$ in Fig. 2 (c) would filter only a part of the radiation from source 2 away, and hence a complete filtering would not occur.

We now apply the theory presented in Section 2 to separate the radiation of source 2 from that of source 1 from the known sum field of the two sources. Once the radiation pattern of source 2 is first obtained, this is then subtracted from the total field to obtain the pattern of source 1. For comparison, we also consider the result in the case where the pure filtering of the undesired coefficients with $n>15$ is performed on the spectrum shown in Fig. 2 (c). Hence, in this comparison, the field of source 1 is estimated using the known spherical vector wave coefficients of the sum field by using (1) with $N=N_{1}=15$. The results for the obtained co-polar directivity after using the pure filtering technique are 
compared in Fig. 3 (a) to that of the correct co-polar directivity of source 1. The results clearly show that the pure filtering technique cannot separate the radiation of source 2 from that of source 1 completely. The errors in the co-polar directivity for the pure filtering technique and the technique introduced in this paper as compared to the correct co-polar directivity of source 1 are shown in Fig. 3 (b). The technique presented in this paper can separate the radiation of source 2 from that of source 1 completely. The insignificantly small ripples in the curve are likely due to numerical inaccuracies in the calculation of the translation coefficients.

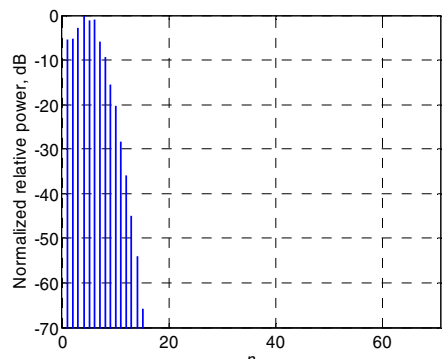

(a)

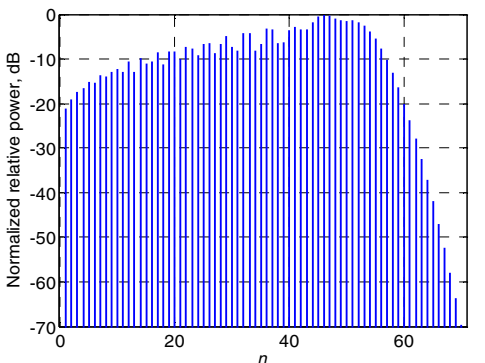

(b)

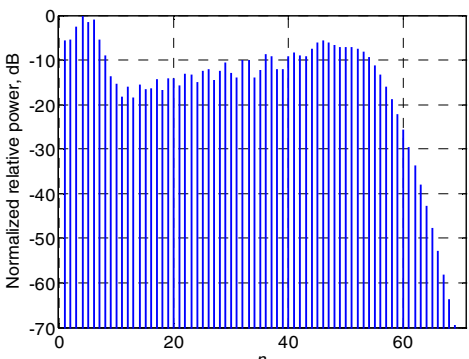

(c)

Fig. 2. The spherical $n$-mode power spectra of the field of source 1 (a), source 2 (b) and that of the total field (c) all in unprimed coordinate system.

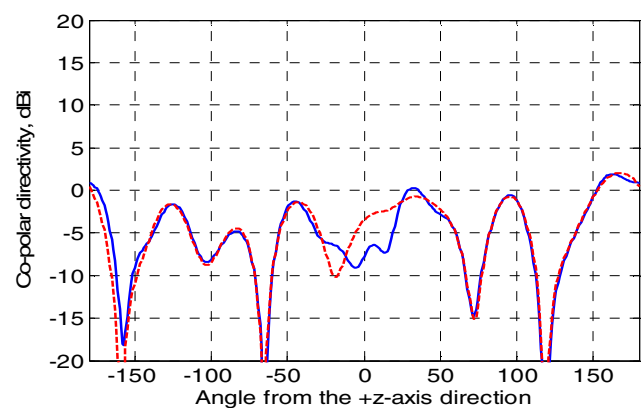

(a)

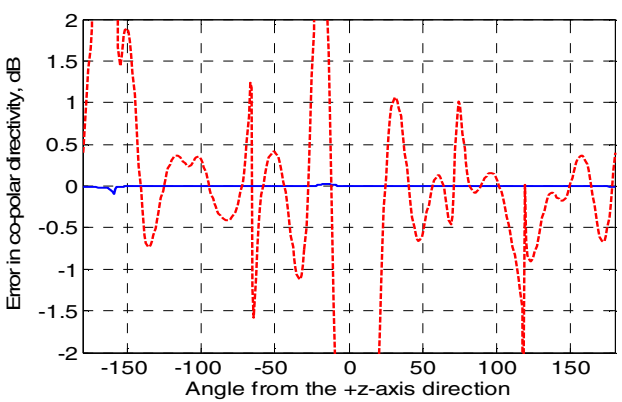

(b)

Fig. 3. Comparison of the correct co-polar directivity to that of the estimated co-polar directivity of source 1 using the pure filtering technique (a). The errors in the co-polar directivity for the pure filtering technique (dashed line) and for

the technique introduced in this paper (solid line) as compared to the correct co-polar directivity of source 1 (b).

\section{Conclusion}

A method for complete and exact separation of the radiation from two separate sources have been presented and shown to work by computer calculations. Future work consists of experimental verification of the technique, examination of its limits, and possibly extending the technique to be applicable for more complex source structures.

\section{Acknowledgments}

T. Laitinen would like to thank Academy of Finland (decision notification no. 129055) for the financial support of this work.

\section{References}

1. C. Cappellin, "Antenna Diagnostics for Spherical Near-Field Antenna Measurements," PhD thesis, Technical University of Denmark, Sept. 2007, 108 p (+ attached papers).

2. D. Hess, "The isofilter ${ }^{\mathrm{TM}}$ Technique: A method of isolating the pattern of an individual radiator from data measured in a contaminated environment," IEEE Antennas and Propagation Magazine, Vol. 52, No. 1, Feb. 2010, pp. $174-181$.

3. J. E. Hansen (ed.), Spherical Near-Field Antenna Measurements, Peter Peregrinus, Ltd., London, 1988. 